\title{
MODEL ATMOSPHERES FOR NOVAE DURING THE EARLY STAGES
}

\author{
R. Wehrsè \\ P. H. Hauschildt \\ Institut f. Theoret. Astrophysik \\ D 6900 Heidelberg \\ G. Shaviv \\ Physics Department \\ Technion \\ IL 32000 Haifa \\ S. Starrfield \\ and Department of Physics, Arizona State University \\ Tempe, AZ 85287-1504
}

IGPP and Theoretical Division, Los Alamos National Laboratory

\begin{abstract}
Continuum and line blanketing models for the photospheres of novae in the early stages of their outbursts are presented. The expanding envelopes are characterized by a very slow increase of density with decreasing radius which leads to very large geometrical extensions and large temperature differences between the inner and outer parts. The spectra show a large IR excess and a small Balmer jump which may be either in absorption or in emission. For the parameters considered $\left(T_{\text {eff }}=10^{4}, 1.5 \times 10^{4}, 2 \times\right.$ $10^{4} \mathrm{~K}, R=10^{11} \mathrm{~cm}$, solar composition), most lines are in absorption. The effects of both modifications in the temperature structure (e.g. by heating from shock fronts) and changes in the abundances of the heavy elements on the emergent spectra are briefly discussed.
\end{abstract}

\section{INTRODUCTION}

The spectra of novae contain a wealth of information on the temperature and density distribution in the ejected material, the elemental abundances in the outer layers, and also the expansion velocity. In addition, as we shall show in Section III, their spectra also provide information on the geometrical extension, luminosity, and the distance. It is evident that the reliable determination of all of the parameters available from spectroscopic analyses would provide significant insight into the physics of the explosion and considerably constrain the range of hydrodynamic models.

In recent years a large amount of high quality spectral information, from X-ray to IR and radio wavelengths, has been obtained for novae in outburst (for reviews see e.g. the contributions by Cassatella, Evans, Gehrz, Hjellming, Ögelman, and Starrfield in these proceedings). However, the analyses of these data, in particular for the early stages of the outburst when the envelope is still optically thick, are still very rudimentary. There are many spectroscopic features of the evolution of novae that are still unexplained and, in addition, there are strong lines in the ultraviolet that have yet to be identified. These problems are compounded because the techniques developed to analyze normal stars, 


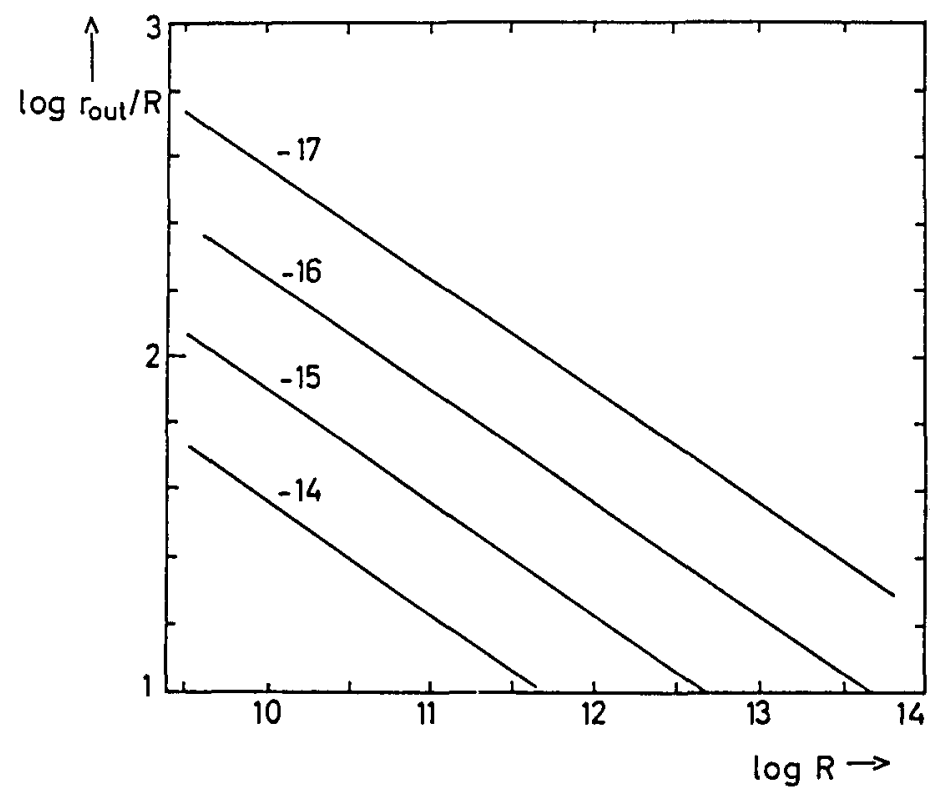

Figure 1. The relation between the outer radius $r_{\text {out }}$ where the optical depth scale starts and the radius $R$ where the optical depth in scattering is unity for different values of the density at $r_{\text {out }}$. The volume between $r_{\text {out }}$ and $R$ is directly accessible by spectroscopy since photons from it can reach the observer without interaction. It is seen that this volume is very much larger than the opaque core region.

whose atmospheres are in hydrostatic equilibrium, can hardly be used on novae since novae atmospheres have very different density and temperature structures from normal stars.

Therefore, in order to analyze the expanding atmospheres of novae, we have developed new methods that are appropriate to their structure. In this paper, we present our first results for blanketed, expanding, model atmospheres and calculate synthetic spectra for novae during their early stages. These atmospheres will ultimately allow detailed analyses of the observed energy distributions and improve the accuracy of the elemental abundances determined from the observations. We note that this method is completely independent from the method normally used to determine elemental abundances in novae: nebular analysis of the emission lines at late stages in the outburst. Comparison of the results, from applying both methods of analysis to the same nova, will provide a unique and important check on each technique.

The atmospheric models are based on the idea (Bath and Shaviv, 1976) that, during the early phases, the outer layers of novae in outburst behave like steady-state, spherical, expanding winds. Energy distributions based on this idea were calculated by Harkness (1983). However, his calculations were restricted to the continuum and the 
radial temperature distributions were kept fixed, i.e., he did not iterate to fulfill the energy equation. He was able to demonstrate the effects of the very low density in the outer layers which resulted in a dominance of scattering and a very large geometrical extension. However, because of the limitations mentioned, the calculated energy distributions cannot be used for spectroscopic analyses. No additional atmospheric modeling of this type for novae is known to us.

In the next Section we list the assumptions made and outline the methods used for construction of our models. Since these atmospheres are very different, not only from normal stars but also from supernovae, several general properties are presented and discussed in Section III. In Section IV we present synthetic spectra and Chapter V is devoted to a discussion. We end with Section VI which describes future improvements that are planned to treat the problems of novae.

\section{MODEL ASSUMPTIONS AND CONSTRUCTION}

Following the ideas of Bath and Shaviv (1976) we consider nova photospheres to be spherical and stationary configurations. Therefore, we assume that all time dependent terms in the hydrodynamic and the radiative transfer equation can be neglected and all quantities depend only upon the radial coordinate (except the specific intensity of the radiation field which depends, in addition, on the angle to the normal direction). In addition, we assume radiative equilibrium, so that energy is transported to the outside only by radiation. Using the results from hydrodynamic calculations of the consequences of thermonuclear runaways in accreted envelopes on white dwarf stars (Starrfield, 1989), we assume that the density varies according to a power law, $\rho \propto \rho_{0}(r / R)^{-n}$, and the expansion velocity is given by $v=\dot{M} / 4 \pi r^{2} \rho$, with $\dot{M}$ being the mass loss rate.

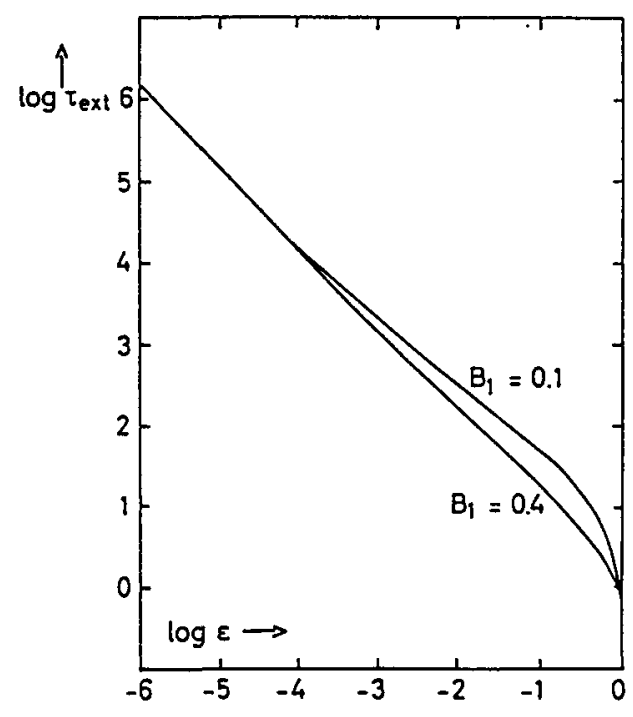

Figure 2. The optical depth in extinction where the local emissivity equals the emergent flux as a function of the ratio $\epsilon=$ absorption coefficient / extinction coefficient for two values of the gradient of the Planck function. 
The models are characterized by the following parameters:

(i) the radius $\mathrm{R}$, which refers to the radius where either the optical depth in absorption at $5000 \AA$ is unity or the optical depth in extinction at $5000 \AA$ is unity,

(ii) the effective temperature $\mathrm{T}_{\text {eff }}$, which is defined by means of the luminosity, $\mathrm{L}$, and the appropriate radius $\left(\mathrm{T}_{\mathrm{eff} f}=\left(L / 4 \pi R^{2} \sigma\right)^{1 / 4}\right.$ with $\sigma=$ Stefan's constant),

(iii) the density parameter, $n$,

(iv) the mass loss rate, $\dot{M}$.

(v) the density, $\rho_{\text {out }}$, at the outer edge,

(vi) the line scattering parameter (see below),

(vii) the elemental abundances.

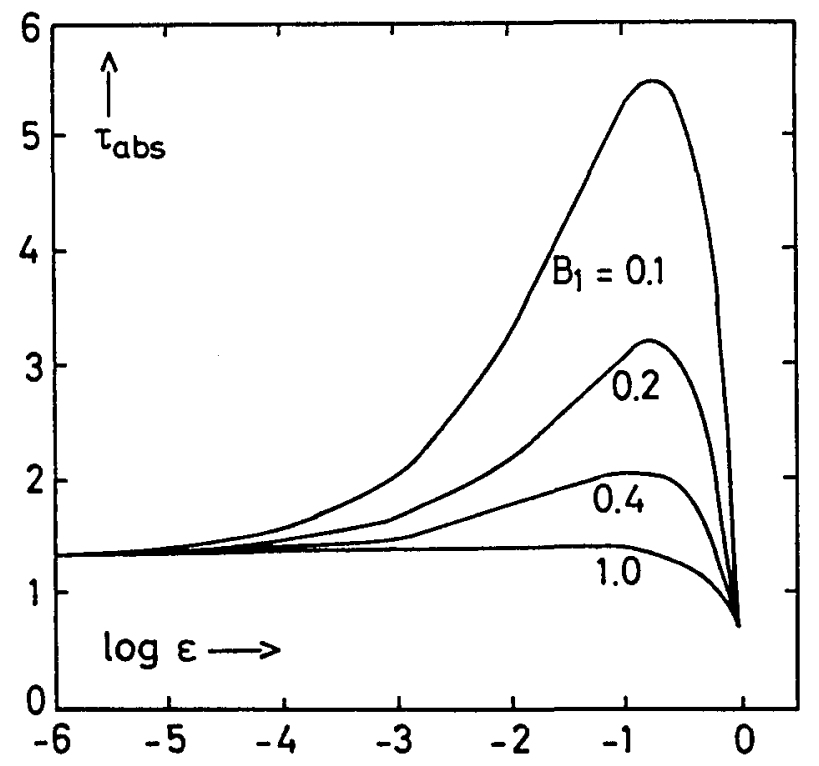

Figure 3. The optical depth in absorption where the local emissivity equals the emergent flux as a function of $\epsilon$. Note that the vertical axis is not logarithmic as in Figure 2 but linear.

Note that as a consequence of the extremely slow increase of density with decreasing radius in novae atmospheres (see below), the density $\rho_{\text {out }}$ is an important parameter in contrast to the situation in supernovae or red giant photospheres.

For the calculation of the spectra we proceed essentially in the same way as we have done for supernovae photospheres (Hauschildt et al. 1989), we solve self-consistently for the radius and the energy equation. We solve the transfer equation in the observer's frame and take all important absorbers, as well as Rayleigh and Thomson scattering, 
into account. In the models with blanketing, we include about 32000 lines selected from the list of Kurucz and Peytremann (1975). Since we are not in a position to calculate the NLTE rate equations for all the levels involved, we assume that all occupation numbers are given by the Boltzmann statistics using the local kinetic temperature. In addition, in order not to neglect the scattering of photons in lines, we divide the line extinction coefficient $\kappa_{l}$ into a fraction $\alpha \kappa_{l}$ that describes the absorption processes and a fraction $(1-\alpha) \kappa_{l}$ that refers to the scattering. We take the same value of $\alpha$ for all lines and consider it to be a free parameter.

The main difference between this technique and our treatment of supernovae photospheres is the replacement of the discrete space formalism (Peraiah 1984) by the matrix-exponential method (Schmidt and Wehrse, 1987) for the solution of the radiative transfer equation. We have also made significant refinements to the temperature correction procedure so that convergence can be achieved for extremely extended configurations.

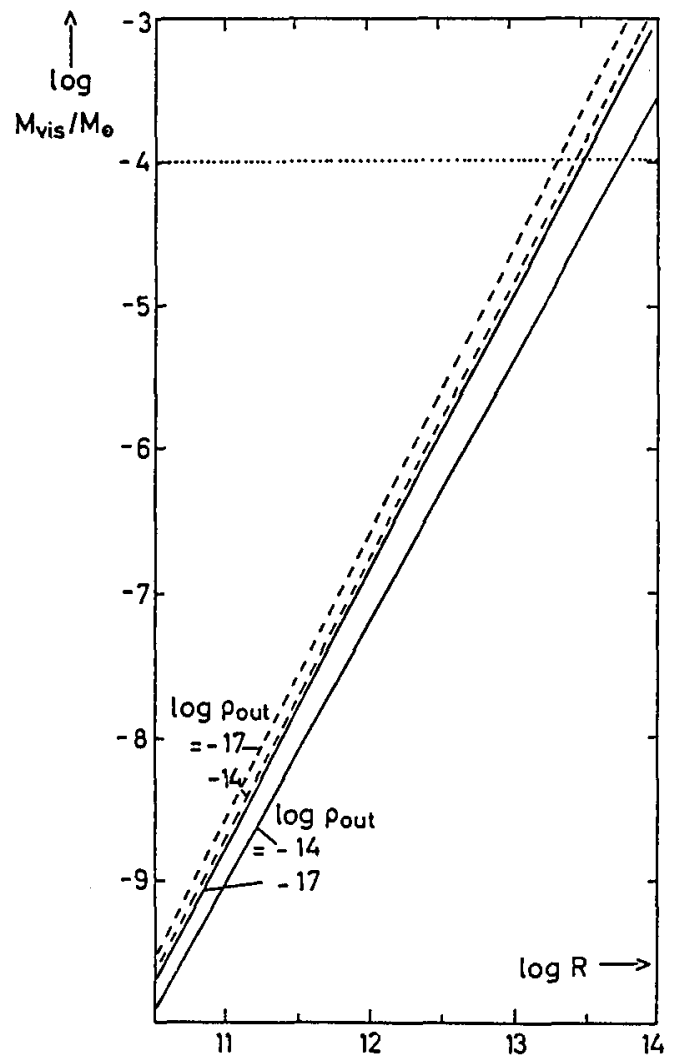

Figure 4. The mass $M_{\text {visible }}$ visible to an observer for two values of the outer density. The full curves refer to an optical depth in scattering of unity, i.e. the volume between $r_{\text {out }}$ and $R$, and the broken curves indicate masses up to the scattering optical depth $300(\approx$ the maximum optical depth from where a photon can emerge without absorption). 


\section{GENERAL PROPERTIES OF NOVA PHOTOSPHERES}

The density exponent, $n$, in nova photospheres is only $\sim 2 \ldots 3$ (Starrfield, 1989), as compared to $n \sim 5 \ldots$ i2 for the atmospheres of SNe II. This has a number of important consequences:

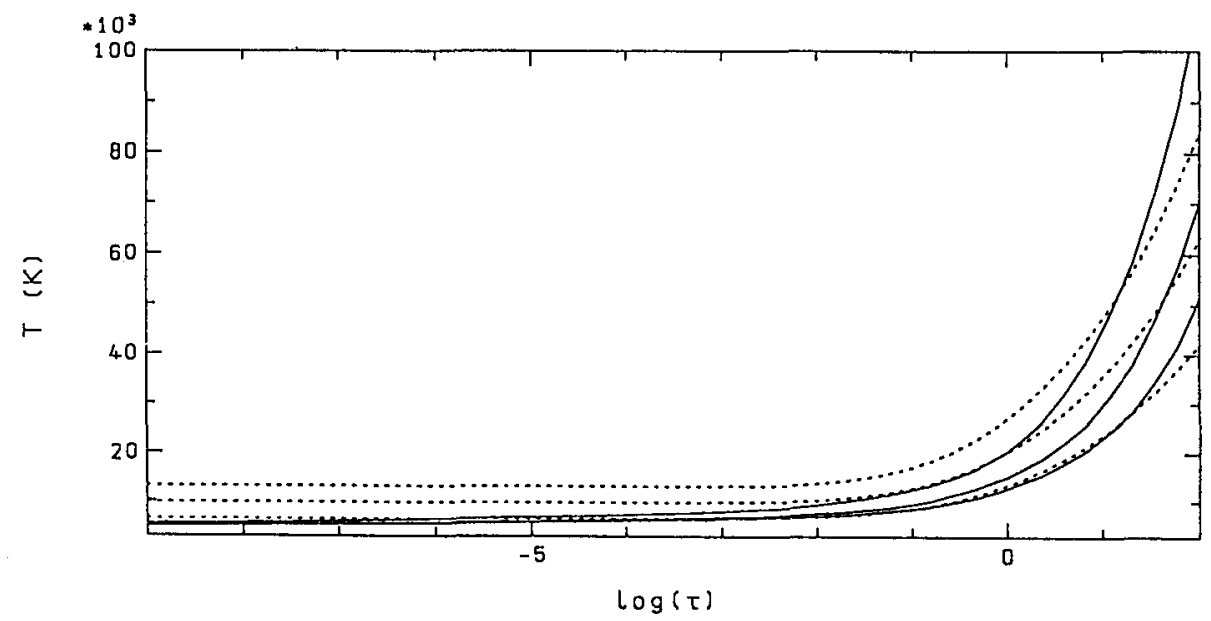

Figure 5. The temperature distributions in nova photospheres with $R_{\left(\tau_{a b}=1\right)}=10^{11}$ $\mathrm{cm}$, outer densities $\rho_{\text {out }}=2 \times 10^{-15} \mathrm{~g} \mathrm{~cm}^{-3}$ and effective temperatures $\mathrm{T}_{\text {eff }}=10^{4}, 1.5 \times$ $10^{4}$, and $2 \times 10^{4} \mathrm{~K}$. Also plotted are the corresponding grey distributions (dotted curves).

(i) nova photospheres are far more geometrically extended (see Figure 1) than those of any other object known to us so that the curvature term in the transfer equation is of great importance;

(ii) the densities are so low that, in large parts of the atmosphere, electron scattering is the main source of opacity and photons generated from thermal emission at rather large depths can reach the surface (possibly after a number of scatterings). In order to demonstrate this effect we used our matrix-exponential code (Schmidt and Wehrse, 1987) to calculate the structure of a plane-parallel atmosphere with a constant ratio of $\epsilon$ (where $\epsilon=$ absorption coefficient / extinction coefficient) and a linear Planck function $B(\tau)\left(B(\tau)=B_{0}+B_{1} \tau\right)$. We determined the optical depth, $\tau_{\text {extinction }}^{0}$, at the point where the emergent flux equals twice the local emissivity (The factor 2 results from the angle integration). The results show (Figure 2) that $\tau_{\text {extinction may, in fact, become }}^{0}$ very large, i.e. photons can arise from very deep layers where the temperature is high if $\epsilon$ is small. Note, however, that for absorbing atmospheres $\tau_{\text {extinction }}^{0}$ is always $2 / 3$ independent of the value of $B_{1}$. Since the corresponding optical depth in absorption (Figure 3) may be larger than unity, these results imply that the color temperatures of scattering dominated, extended, atmospheres can be very high; 
(iii) the mass depth $\mathrm{M}_{\text {visible }}$ from which photons can escape (that part of the atmosphere directly visible to an outside observer) may be rather large. As is seen in Figure 4, it can reach an appreciable fraction of the total accreted mass of $\sim 10^{-4} M_{\odot}$ to $\sim 10^{-5} M_{\odot}$ (Starrfield, 1989);

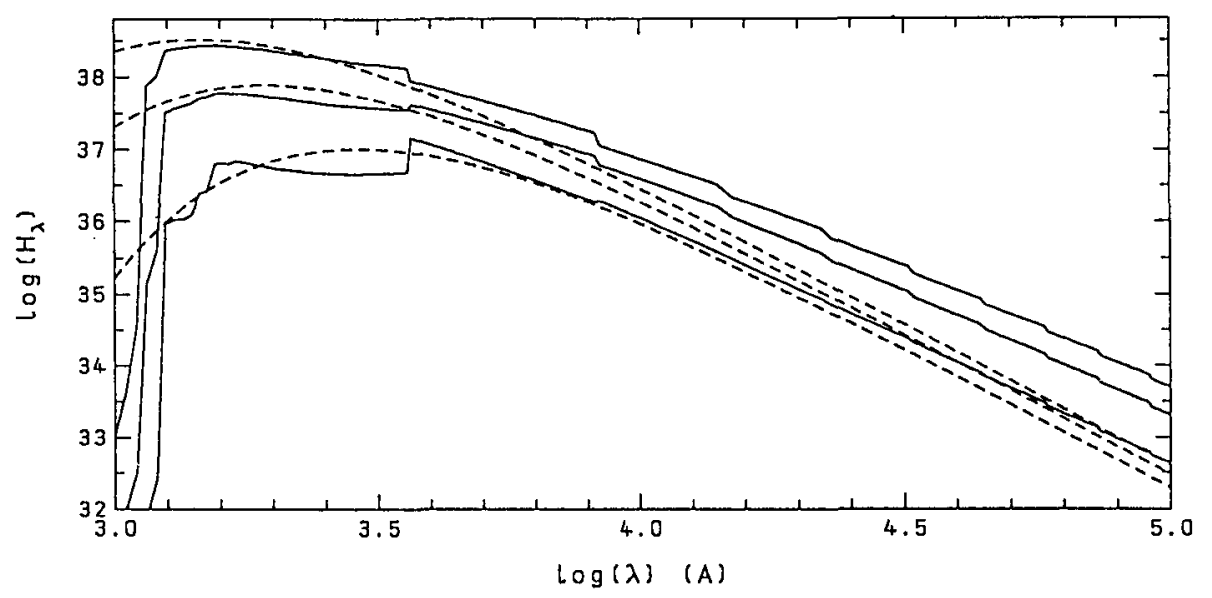

Figure 6. Continuum fluxes of nova photospheres (full curves) with $R_{\left(\tau_{a b}=1\right)}=10^{11}$ $\mathrm{cm}$, outer densities $\rho_{\text {out }}=2 \times 10^{-15} \mathrm{~g} \mathrm{~cm}^{-3}$ and effective temperatures $\mathrm{T}_{\text {eff }}=10^{4}, 1.5 \times$ $10^{4}$, and $2 \times 10^{4} \mathrm{~K}$ from the ultraviolet to the infrared range. The broken lines give black body fluxes for the same temperatures.

(iv) the temperature distributions (Figure 5) are characterized by optically thin (in absorption) outer parts in which the temperature $\mathrm{T} \propto r^{-1 / 2}$ (as a consequence of the geometrical dilution) and inner parts where the temperature rises very fast. This important result implies that we can have a large number of ionization stages simultaneously present in the spectrum. In addition, if the outer density is low enough, molecules and perhaps even dust can form in the outer layers as is observed (see e.g. Gehrz, 1988). We note that the observations of multiple ionization stages in novae spectra is one of the important diagnostics of these spectra and its cause has been, heretofore, unexplained.

\section{SYNTHETIC SPECTRA}

In Figs. 6 and 7 we show energy distributions for models in which only continuous absorption and scattering is taken into account. The parameters are: $\mathrm{T}_{\text {eff }}=10^{4}, 1.5 \times$ $10^{4}$, and $2 \times 10^{4} \mathrm{~K}, R_{\left(\tau_{a b}=1\right)}=10^{11} \mathrm{~cm}, \rho_{\text {out }}=2 \times 10^{-15} \mathrm{~g} \mathrm{~cm}^{-3}, \dot{M}=10^{-7} M_{\odot} y^{-1}$ leading to an maximum expansion velocity of $v=1000 \mathrm{~km} \mathrm{~s}^{-1}$.Solar composition and an $\alpha$ parameter of 0.5 are assumed. While we are fully aware that nova abundances are far from solar (Truran and Livio 1986), we regard these calculations as our baseline 
study to which all further work will be compared. It is seen that in the IR range all our atmospheres have a strong excess over the equivalent black-body distribution plus a very flat energy distribution which is caused by the large extension. It cannot be interpreted as direct evidence for material with the corresponding color temperature in the atmosphere. The Paschen and the Balmer jumps are both quite weak and can be in emission or absorption as was shown earlier by Harkness (1983). This behavior is caused by the flux per unit surface area on the short wavelength side of the jump being smaller than that on the long wavelength side (since it is formed in the outer and cooler layers), however, this effect may also be partially compensated for or even overcompensated for by the larger emitting area. The Lyman edge is always in absorption because the steep gradient of the Planck function at $912 \AA$ always prevails over the extension of the atmosphere.

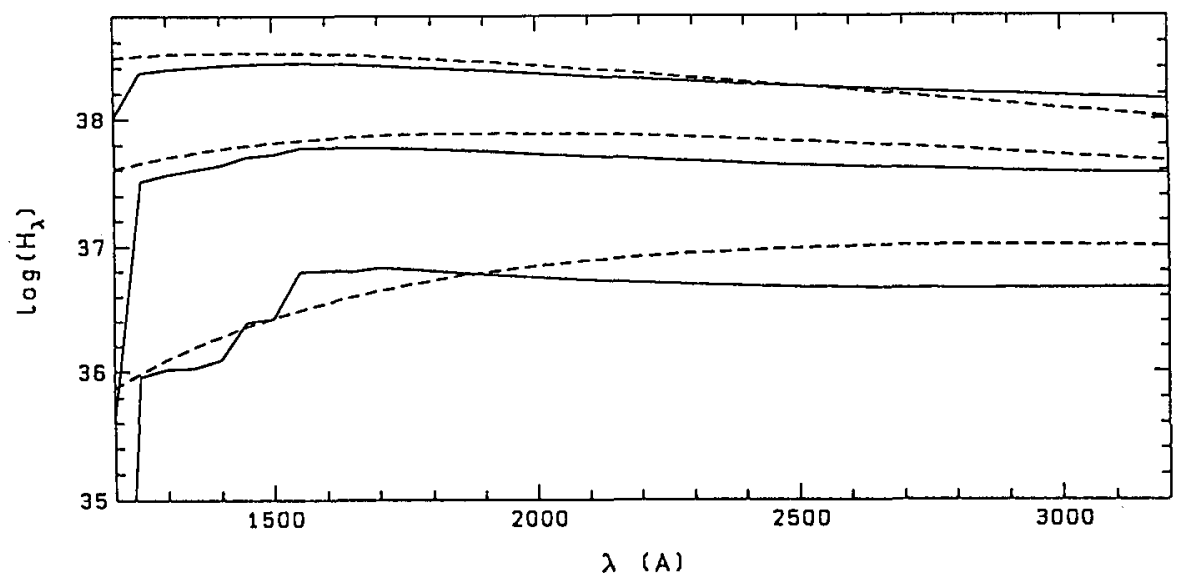

Figure 7. Same as Figure 6 but on an extended linear scale for the UV range.

In the UV we see, in addition, the presence of the absorption edges of neutral species (CI, MgI, SiI etc.) even for very high $\mathrm{T}_{\text {eff }}$. This is caused by the presence of these species in the outer cooler layers. In the inner parts of the atmospheres, the radiation field is very high at these frequencies. This implies that a CII region, which possibly surrounds the atmosphere, must be very small or ionized by other sources (such as, for example, shock fronts).

For the parameters under discussion here, there is very little effect on the temperature structure or the run of the continuum when we include of lines (Figure 8). All lines appear to be still in absorption since the Planck function drops off rapidly (which can also be deduced from the very small residual fluxes). Although, in this paper, we did not check the contributions of specific transitions, it seems to us that essentially all 
observed features found in the UV spectra obtained with the IUE Satellite are severe blends. Finally, the line profiles for novae spectra are quite narrow as compared to those for supernovae spectra since the spread of expansion velocities within the atmosphere is much smaller. However, they are, obviously, much broader than found for normal stars with the same $\mathrm{T}_{\text {eff }}$.

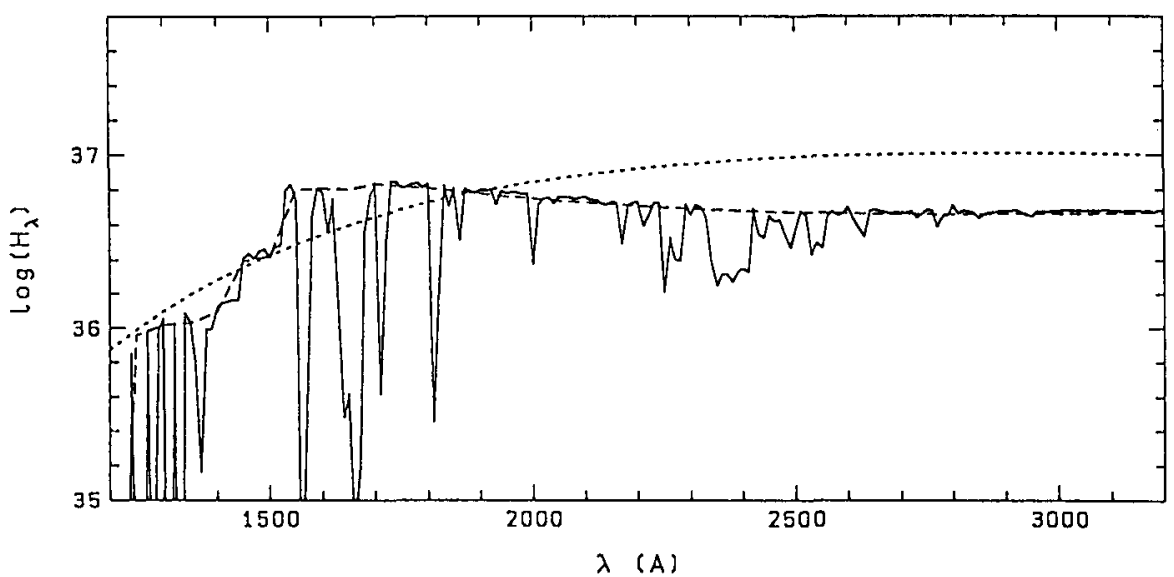

Figure 8. Comparison of the fluxes of a continuum model (broken curve) with a radius $R\left(\tau_{a b s}=1\right)=10^{11} \mathrm{~cm}$, an outer density $\rho_{o u t}=2 \times 10^{-15} \mathrm{~g} \mathrm{~cm}^{-3}$ and an effective temperature $\mathrm{T}_{\text {eff }}=10^{4} \mathrm{~K}$, of a metal line blanketed model (full curve) with the same parameters, and of a black body (dotted line) of the same temperature.

\section{DISCUSSION}

The spectra presented here have been calculated using the assumptions of radiative equilibrium and solar composition which are both questionable for novae. We note that during the explosion it seems reasonable that shocks can form and heat the outer layers by non-radiative processes. It is also clear that most novae show strong evidence for enhanced abundances of the CNONeMg elements (Truran and Livio 1986; Starrfield 1989, Sparks et al. 1987).

In order to investigate possible consequence of deviations from radiative equilibrium, we calculated, in addition, a model in which the temperature for layers $\tau_{a b s}<10^{-4}$ was kept (artificially) constant. As is seen in Figure 9, the line spectrum changes dramatically. There is a significant change in the shapes and strengths of many features and some go strongly into emission although the relative change in the total flux is only $\sim 10^{-3}$. This result implies that the occurrence of emission lines in observed spectra indicates either that the continuum has become weak (i.e. the line forming region is very 
much larger than the continuum forming region) or that the temperature in the outer regions is enhanced by the dissipation of non-radiative energy. With good models it should be possible to derive the relative importance of these effects from the flux ratios.

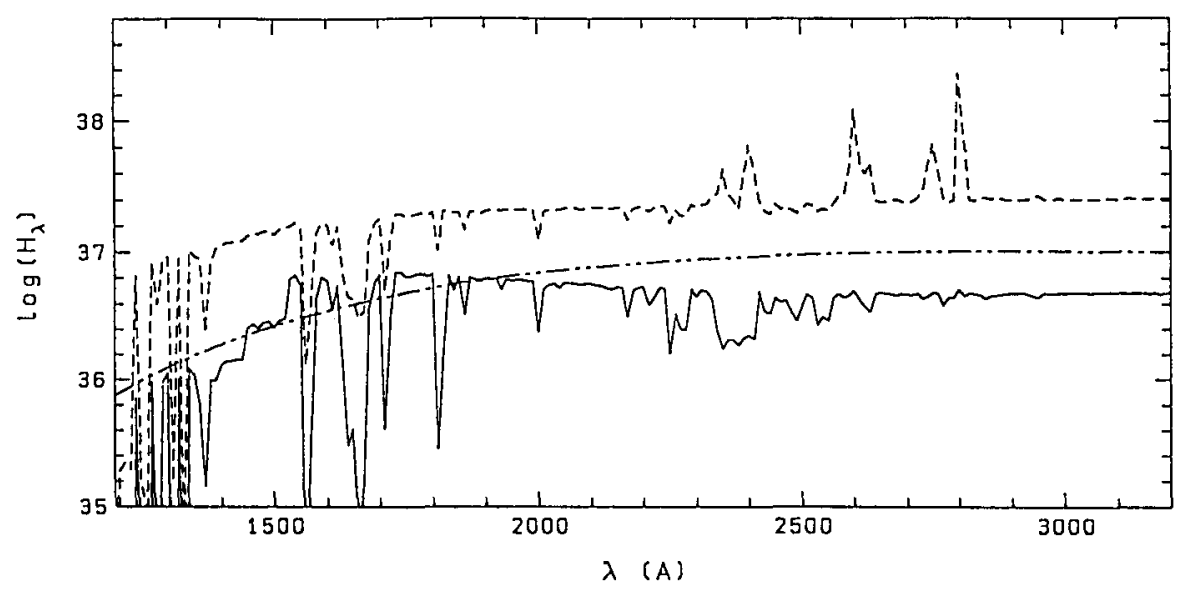

Figure 9. Demonstration of the significance of the temperature distribution in the outer layers: The full curve shows the same blanketed energy distribution as Figure 8 for which the temperature distribution of Figure 5 is used for all optical depths whereas for the broken curve it is assumed that the temperature distribution follows this run only up to $\tau_{a b s}<10^{-4}$ and is constant for the upper layers.

In order to demonstrate the effects of composition changes on the spectra, we have calculated an additional model in which the abundances of heavy elements was increased by 0.7 dex. Figure 10 shows that the overall structure of the spectrum remains unchanged. However, individual features can appreciably change their strengths and line shapes due to the very complex competition between the $\mathrm{B}$ gradient and the extension of the atmosphere (see above). This causes some lines to again go into emission. It is important to realize that changing the elemental abundances severely affects the lines and that it should be relatively straightforward to obtain abundances from analyses of observed spectra using models such as we have presented in this paper.

Finally Figure 11 shows a comparison between the UV spectrum of an observed nova (LMC nova 1988 no. 1) and one of the models discussed here. Note that no attempt to fit the observed spectrum was made. The overall agreement in the continuum is quite well, but it seems that the model contains too few lines to reproduce all the features for $\lambda>2600 \AA$. 


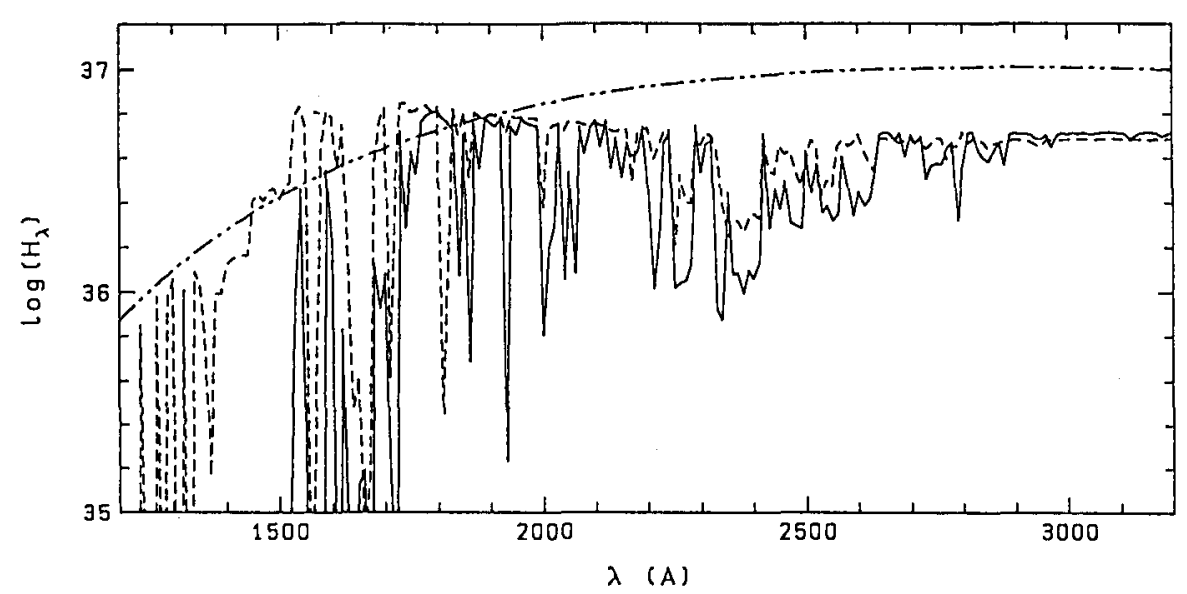

Figure 10. Effects of abundance changes on the UV spectra of novae: The broken curve shows the same blanketed flux distribution as Figure 8 whereas for the full curve an overabundance of all heavy elements by a factor of 5 is assumed. The dotted line again gives the black body spectrum.

\section{FUTURE DEVELOPMENTS}

Model atmospheres, such as those presented here, make a quantitative interpretation of early novae spectra possible for the first time. An extensive program of comparing observed and computed spectra is presently under way by our group. However, it is also evident that in the future the following improvements will have to be introduced:

(i) a full NLTE treatment of ions like HI, HeI, HeII, CII, CIII, NII, NIII, FeII, FeIII, etc. which will improve the accuracy of abundances derived from subordinate transitions and from lines which are formed very close to the surface;

(ii) spatially resolved images of nova envelopes often show significant deviations from spherical symmetry so that 2 or even 3 dimensional modeling of the density, temperature, and radiation fields seems to be required. Such modeling is certainly feasible by means of powerful present day computers (see, for example, Stenholm et al. 1989) and should be tried. On the other hand, it is not clear to what extent unique values for nova parameters can be derived so that many fundamental aspects may have to be rediscussed in order to fully understand the results;

(iii) both direct images and high resolution spectra (Krautter, 1988) demonstrate that nova shells are in most cases not as homogeneous as assumed in the models but that, instead, there are inhomogeneities (knots, blobs etc.) of very different scales and contrasts. Therefore, a statistical treatment would be most appropriate. A straightforward generalization of the approach developed by Gierens et al. (1986) should not only give a better representation of reality but, in addition, it should be possible to obtain 


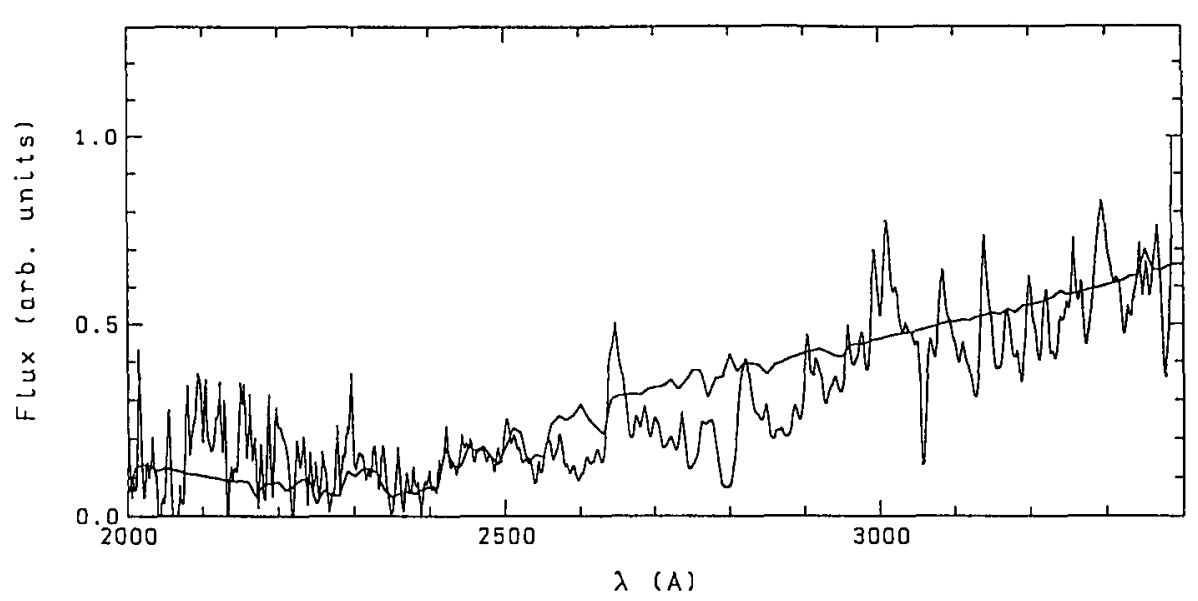

Figure 11. Comparison of the UV spectrum of the LMC nova 1988 no. 1 (Mar 29,1988) (thin line) and a line-blanketed model with the parameters $\mathrm{T}_{\text {eff }}=10^{4} \mathrm{~K}, R_{\tau_{a b}=1}=$ $10^{11} \mathrm{~cm}, \rho_{\text {out }}=2 \times 10^{-15} \mathrm{~g} \mathrm{~cm}^{-3}$ and solar abundances (thick line).

the information contained in the local minima and maxima of line profiles and to have much improved estimates of the errors in the derived parameters.

S. Starrfield is grateful to Drs. S. Colgate, A. N. Cox, C. F. Keller, M. Henderson, and K. Meyer for the hospitality of the Los Alamos National Laboratory and a generous allotment of computer time. This work was supported in part by NSF Grants AST8516173 and AST88-18215 to Arizona State University, by the Institute of Geophysics and Planetary Physics at Los Alamos, by NASA grants to Arizona State University (NAG5-481) and to the University of Colorado (NAS5-28731), and by the DOE.

\section{REFERENCES}

Bath, G.T, Shaviv, G.: 1976, Monthly Notices Roy. Astronom. Soc. 197, 305.

Gehrz, R.D.: 1988, Ann. Rev. Astron. Astrophys. 26, 377.

Gierens, K.M., Traving, G., Wehrse, R.: 1987, Journal Quant. Spectroscop. Radiat. Transfer, 37, 361.

Harkness, R.P.: 1983, Monthly Notices Roy. Astronom. Soc. 204, 45.

Hauschildt, P.H., Shaviv, G., Wehrse, R.: 1989, Astron. Astrophys., 210, 262.

Krautter, J.: 1988, in A Decade of UV Astronomy with the IUE Satellite, Vol. 1, p. 153. Kurucz, R.L., Peytremann, E.: 1975, A Table of Semiempirical gf Values, Smithsonian Astrophysical Observatory, Special Report 362.

Peraiah, A.: 1984, in Methods in Radiative Transfer, Kalkofen, W., Ed., Cambridge University Press, p. 341.

Schmidt, M., Wehrse, R.: in Numerical Radiative Transfer, Kalkofen, W., Ed., Cambridge University Press 
Sparks, W.M., Starrfield, S., Truran, J.W., 1987: in RS Ophiuchi, Bode, M.F., Ed., VNU Science Press, Utrecht, p. 39.

Starrfield, S.: 1989, in Classical Novae, Bode M.F., Evans A., Eds., Wiley, p. 39.

Stenholm, L.G., Störzer, H., Wehrse, R.: 1989, J. Quant. Spectroscop. Radiat. Transfer, submitted.

Truran, J. W., and Livio, M.: 1986, Astrophys. J., 308, 721. 